\title{
Strategies De Preservation Des Forêts Villageoises Dans Le Departement D'akoupe Au Sud-Est De La Cote d'Ivoire
}

\begin{abstract}
Dagou Ahosso Franck Olivier,
Université Félix Houphouët-Boigny, Abidjan, Côte d'Ivoire, Institut d'Ethno-Sociologie, Département de Sociologie. Laboratoire d'Etude et de Recherches Interdisciplinaires en Sciences Sociales et Humaines (LERISSH)
\end{abstract}

Doi:10.19044/esj.2020.v16n7p10 ～URL:http://dx.doi.org/10.19044/esj.2020.v16n7p10

\section{Résumé}

Les pratiques de l'agriculture itinérante incontrôlée, en Côte d'Ivoire en général et dans le Département d'Akoupé en particulier, ont eu des effets néfastes sur le couvert forestier. La présente étude, a pour objectif de décrire les stratégies que développent les acteurs du domaine forestier dans la préservation des forêts villageoises. La méthode adoptée a consisté à combiner recherches documentaires, observations directes et enquêtes de terrain. Les enquêtes ont été conduites sur la base d'un guide d'entretien. Pour rendre les résultats objectifs, la théorie de l'interactionnisme symbolique et celle de la représentation sociale ont été adoptées. Les résultats ont montré que l'Etat de Côte d'Ivoire et les populations locales développent des stratégies tant modernes que traditionnelles, à travers les structures chargées du domaine forestier, pour préserver les forêts villageoises.

Mots-clés : Agriculture itinérante, Stratégie, Préservation, Forêt villageoise, Côte d'Ivoire 


\title{
Preservation Strategies of Village Forests in the Departement of Akoupe in the South East of Ivory Coast
}

\begin{abstract}
Dagou Ahosso Franck Olivier,
Université Félix Houphouët-Boigny, Abidjan, Côte d'Ivoire, Institut d'Ethno-Sociologie, Département de Sociologie. Laboratoire d'Etude et de Recherches Interdisciplinaires en Sciences Sociales et Humaines (LERISSH)
\end{abstract}

\begin{abstract}
The uncontrolled agricultural nomadic practices conducted in Ivory Coast, especially in the Department of Akoupe, have resulted to some negative impacts on the forest covert. This investigation conducted in that department targets the description of the strategies implemented by the actors of the forest domain, who are willing to protect the natural forests in villages. The methodology they adopted consisted of a combination of documentary researches, direct observations, and ground investigations. The investigations occurred on the basis of an interview guide targeting the performers of the forest area. To be much more objective, the theory of the symbolic interactionism and that of the social representation were adopted. The results of the study show that the Ivorian government as well as the local populations, using the structures in charge of the forest area, created both modern and traditional strategies to preserve the village forests.
\end{abstract}

Keywords: Nomadic agriculture, strategies, preservation, village forests, Ivory Coast

\section{Introduction}

Le BNETD (Bureau National D'Etudes Techniques et de Développement) a évalué la couverture forestière de la Côte d'Ivoire à 6,38 millions d'hectares en 2005. Cette forêt était estimée à 16 millions d'hectares dans les années 1900 (Chatelain et al.,2004). Cette évaluation faite par le BNETD est composée de 4,2 millions d'hectares de forêts classées (constituées de plantations forestières, industrielles et réserves biologiques) et de 2,18 millions d'hectares d'aires protégées (sous forme de parcs nationaux et de réserves). Aujourd'hui, la zone forestière est réduite à quelques lambeaux de forêts secondaires au sein d'une succession de plantations villageoises ou 
industrielles et de jachère. René Dumont et Charlotte Paquet (1991) avaient tiré la sonnette d'alarme quand ils évoquent l'état de dégradation de la forêt en Côte d'Ivoire. Ils affirmaient qu'il ne restait plus, dans la zone humide, de vrais massifs forestiers mais des boisements limités et encore, souvent écrémés, des arbres de valeurs moins troués et mités d'éclaircies partielles ou totales.

En 2014, les statistiques de la Banque Mondiale ont montré que le secteur agricole constitue le moteur de la croissance de l'économie ivoirienne. Cette économie contribue à créer 22,3\% du PIB puis représente $47 \%$ des exportations globales du pays (62\% hors pétrole). Cette agriculture est dominée par le binôme café-cacao. Selon ces mêmes statistiques, le cacao est cultivé sur une superficie de 2.300.000 d'hectares et a atteint 1,74 millions de tonnes. Ce qui représente $40 \%$ de la production mondiale. Le café est pratiqué sur un espace de 1 million d'hectare. Ces deux cultures représentent, en valeur, les 2/3 des exportations agricoles et occupent à elles seules plus de $75 \%$ des terres arables. À côté de ces deux cultures de rente, il y a le palmier à huile cultivé sur 200.000 hectares et l'hévéa qui occupe une superficie de 300.000 hectares (Ministère de l'Agriculture et du Développement Rural, 2015). En effet, cet accroissement de la production n'est nullement le fait d'un gain de productivité mais plutôt lié à l'accroissance des superficies cultivées.

Le Département d'Akoupé n'est pas en marge de cette croissance de superficies des terres agricoles. Selon les estimations de la SAPH (2014), de 1698,25 hectares d'hévéa en 2008, on est passé à 1798,25 hectares. Ce qui représente une majoration de 102 hectares. En outre, l'ANADER (Agence Nationale pour le Développement Rural) a évalué les superficies de cacao à 14077,92 hectares en 2005 et à 14602,11 hectares en 2014. Concernant le café, il est passé de 132,5 hectares en 2005 à 157,2 hectares en 2014. On note également l'existence des cultures vivrières telles que la banane plantain dont les superficies sont estimées semestriellement. La banane plantain est estimée à 105,05 hectares en 2015 et à 178 hectares en 2016. Quant au manioc, il est évalué à 212,3 hectares en 2015 et à 265,05 hectares en 2016.

L'on déduit donc que les cultures de cacao, de café et de l'hévéa ont fait disparaitre, à elles seules, près de 650,89 hectares de forêts (superficies agricoles utilisées en moins de dix ans dans le Département d'Akoupé (Rapport annuel DDARA Adzopé, 2015-2016). Cette situation de déforestation a suscité la mise en place de stratégies pour la préservation des forêts villageoises du Département d'Akoupé. Dès lors, l'on se pose les questions suivantes:

Quelles sont les stratégies développées par les différents acteurs pour préserver les forêts villageoises dans le Département d'Akoupé ? 
Autrement dit, quelles sont les stratégies de préservation mises en place par l'État et les populations locales du Département d'Akoupé? C'est dans cette dynamique que s'inscrit cette étude.

\section{Méthodologie}

Elle consiste à circonscrire les champs de la recherche à travers le site et la population d'étude, l'échantillon et la méthode d'analyse.

\subsection{Le site et la population d'étude}

\subsubsection{Le site de l'étude}

Cette étude a été réalisée dans le Département d'Akoupé situé dans le Sud-est de la Cote d'Ivoire précisément dans la région de la Mé. Cette localité est située à environ 139 km d'Abidjan, la capitale économique. Il est limité au Sud par la préfecture d'Adzopé, à l'Ouest par la circonscription d'Agboville, à l'Est par la ville d'Abengourou et au Nord par la sous-préfecture d'Arrah (Cf. carte ci-après).

Figure 1. Situation géographique du département d'Akoupé

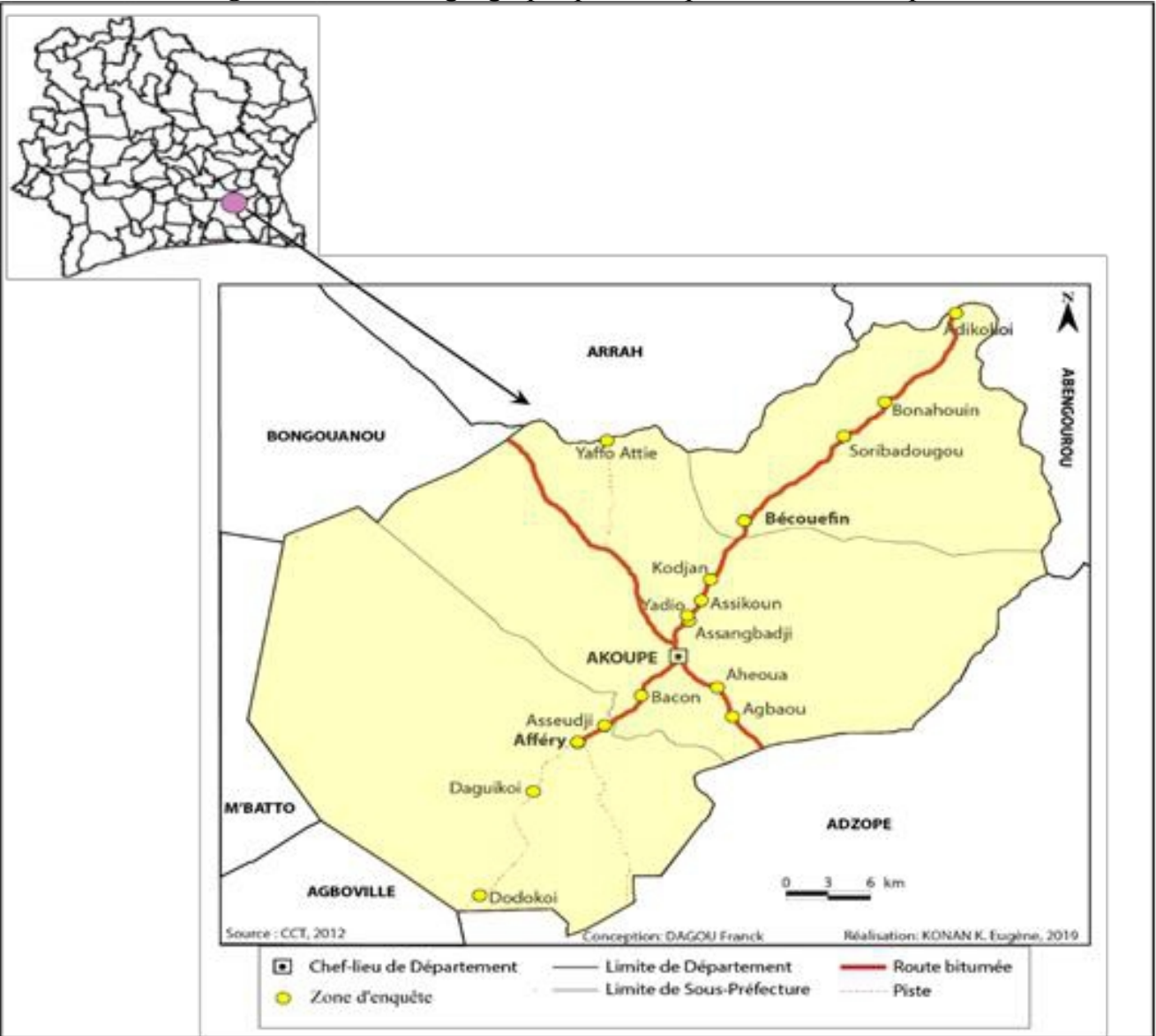

Source : CCT, 2012 


\subsubsection{Population cible}

Cette étude s'est intéressée à différentes catégories d'acteurs sociaux. Le critère de choix a porté sur l'activité socioprofessionnelle. D'abord, les chefs d'exploitations (agriculteurs), personnes ressources en contact directe avec la forêt. Ensuite, la population experte qui a une maitrise avérée ou supposée sur le sujet (préfet, responsable CNRA, Ministère de l'Agriculture, Ministère des Eaux et Forêts, ANADER, ONG GAYA et Coopératives agricoles). Enfin, la population témoin (chefs coutumiers), ces acteurs sont les premiers garants de la tradition et propriétaire terrien.

\subsection{L'échantillon}

La collecte des données a nécessité l'usage d'une approche qualitative et l'option d'un échantillonnage non probabiliste impliquant l'utilisation d'un échantillonnage raisonné. En effet, pour réaliser cette étude, un échantillon a été sélectionné qui parait représentatif de la population cible. Sur cette base, l'échantillon est composé de : 17 chefs d'exploitations (agriculteurs) possédant au moins 5 hectares de forêts, préfecture du département, (1) agent du Ministère de l'agriculture, (1) agent du Ministère des Eaux et Forêts, (1) agent de l'ANADER et (1) responsable de l'ONG GAYA, à cela s'ajoute 2 représentants de coopératives suivis de 5 membres de la notabilité. Au total, la taille de l'échantillon est estimée à 29 personnes.

\subsection{La méthode d'analyse}

L'analyse des données s'est faite selon l'approche compréhensive. Cette approche a permis de décrire les stratégies que chaque acteur (structures étatiques et populations locales) développe pour préserver les forêts villageoises.

\subsection{Perspective théorique}

Pour rendre les résultats objectifs, il a été fait recours à la théorie de la représentation sociale et celle de l'interactionnisme symbolique.

\section{Résultats}

\subsection{Politiques et stratégies de l'État dans la préservation des forêts}

En 1992, la Côte d'Ivoire a signé et ratifié la Convention de Rio sur la diversité biologique. L'objectif officiellement adopté par la communauté lors de ce sommet est que les ressources et les terres forestières doivent être gérées d'une façon écologiquement viable afin de répondre aux besoins sociaux, économiques, écologiques, culturels et spirituels des générations actuelles et futures. Pour mieux préserver son patrimoine forestier destiné à l'activité agricole, l'Etat de Côte d'Ivoire va adhérer, en juin 2011, au mécanisme de REDD+. Cette adhésion vise à contribuer à la lutte mondiale contre le 
changement climatique et surtout à la restauration de sa couverture forestière dégradée.

Dans cette optique, plusieurs actions ont été engagées en vue de promouvoir le développement durable en Côte d'Ivoire. Ces actions sont entre autres les programmes de renforcement du cadre institutionnel et juridique des capacités nationales de gestion durable de l'environnement ; les programmes de sensibilisation, d'éducation et d'informations environnementales privilégiant l'implication appropriée de la société civile (Plan d'Investissement Forestier, 2016).

C'est ainsi que la mission d'encourager les paysans à l'utilisation de l'engrais (comme solution à la préservation des forêts villageoises) est confiée au Ministère de l'Agriculture et du développement rural.

À cet effet, le premier responsable du Ministère de l'Agriculture du département d'Akoupé dit :

"L'engrais constitue l'un des intrants les plus importants pour promouvoir une agriculture durable, respectueuse de l'environnement et capable d'apporter des solutions aux enjeux du changement climatiques. Ce pourquoi nous engageons les paysans à utiliser les engrais. »

Pour honorer tous ces engagements et mettre en pratique toutes les stratégies de préservation des forêts, l'Etat de Côte d'Ivoire a confié des tâches sous forme de missions aux structures d'encadrement telles que l'ANADER (Agence Nationale au Développement Rural). Cette société regroupe l'Etat, le secteur privé, les producteurs, la société civile et leurs organisations professionnelles. Celle-ci a pour mission de responsabiliser davantage les producteurs et leurs organisations professionnelles. Elle est également chargée de conseiller, d'informer, de former et d'encadrer le monde rural.

En somme, elle a pour objet social de contribuer à la promotion du monde rural par la professionnalisation des producteurs agricoles. Pour ce faire, elle inculque aux agriculteurs (paysans) les nouvelles techniques agricoles qui consistent à introduire des arbres dans le paysage agricole. Comme le dit le responsable de cette structure :

"On demande aux paysans d'associer les cultures aux arbres, c'est une technique d'agroforesterie qui contribue à découpler la production agricole et la déforestation afin d'aboutir à une agriculture zéro déforestation ».

S'agissant des destructions de forêt liées à la croissance exponentielle de la demande de terres agricoles, l'ANADER, le REED+ et ses différents partenaires comptent ralentir ce phénomène par l'amélioration de la productivité des cultures via le développement de pratiques agricoles 
intensives à faible impact environnemental. Cette autre opportunité de découpage entre production et déforestation agricole concerne toutes les filières agricoles du pays. Elle concerne non seulement le cacao mais aussi l'hévéa, le café, le palmier à huile, l'anacardier etc. par l'introduction d'arbre dans le paysage agricole. Cette approche concentre les interventions sur les zones agricoles à l'intérieur et autour des terres forestières afin d'examiner l'impact le plus significatif possible. Concernant le phénomène des destructions des forêts lié à la consommation urbaine de bois énergie, de loin la plus importante cause des prélèvements forestiers, l'ANADER et le REED+ comptent ralentir ce phénomène par l'amélioration des filières bois de feu et charbon de bois. Cela est évoqué dans les propos du responsable de l'ANADER :

«Avec nos différents partenaires, on compte mettre en place des plantations d'essences appropriées à croissance rapide pour permettre à la Côte d'Ivoire de réduire la déforestation en milieu rural ».

À cette structure, s'ajoute l'administration forestière (le corps des Eaux et Forêts) qui est également chargée de gérer le domaine rural. En effet, elle demande aux paysans de faire l'agroforesterie dans le cas des plantations de cacao et de café. Elle sensibilise sur le reboisement compensatoire c'est-à-dire le paysan doit planter des arbres sur sa parcelle de terre en fonction du volume coupé. Pour justifier ce qui précède, le chef de cantonnement des Eaux et Forêts du département d'Akoupé s'illustre en ces termes :

«Il est demandé aux paysans de planter des arbres dans leurs différentes plantations. Par exemple pour la culture du cacao, il est conseillé aux cultivateurs de planter dix-huit (18) arbres à l'hectare. Cette technique lui permet de faire le reboisement ».

\subsection{Stratégies développées par les autorités villageoises et administratives du département d'Akoupé dans la préservation des forêts villageoises}

Dans cette partie, il est important de noter qu'il existe deux types de forêts villageoises. Il y a celles qui sont à caractère agricole et celles qui sont sacrées entièrement réservées à l'expression culturelle d'une communauté donnée. Ces deux forêts villageoises sont privées et sont sous la gestion des populations locales.

S'agissant des forêts villageoises qui servent à pratiquer l'activité agricole, les populations villageoises qui en possèdent encore mettent en place des stratégies pour les conserver. C'est ainsi que des lopins de forêts sont laissés sur des parcelles de terre qui servent d'activités agricoles. En effet, ces petites parcelles sont conservées pour les générations futures. 
A titre d'exemple, un chef de famille qui possède 5 hectares, 2 ou 3 hectares sont consacrés aux cultures et le reste est conservé pour les générations futures, c'est-à-dire sa descendance. A ce propos, un chef de famille révèle :

"J'ai hérité de mon père, six hectares de forêt. J'ai utilisé trois pour faire mes plantations et j'ai gardé les trois autres pour mes enfants ».

Photo 1. Défrichement de superficie de forêt pour la mise en place des cultures

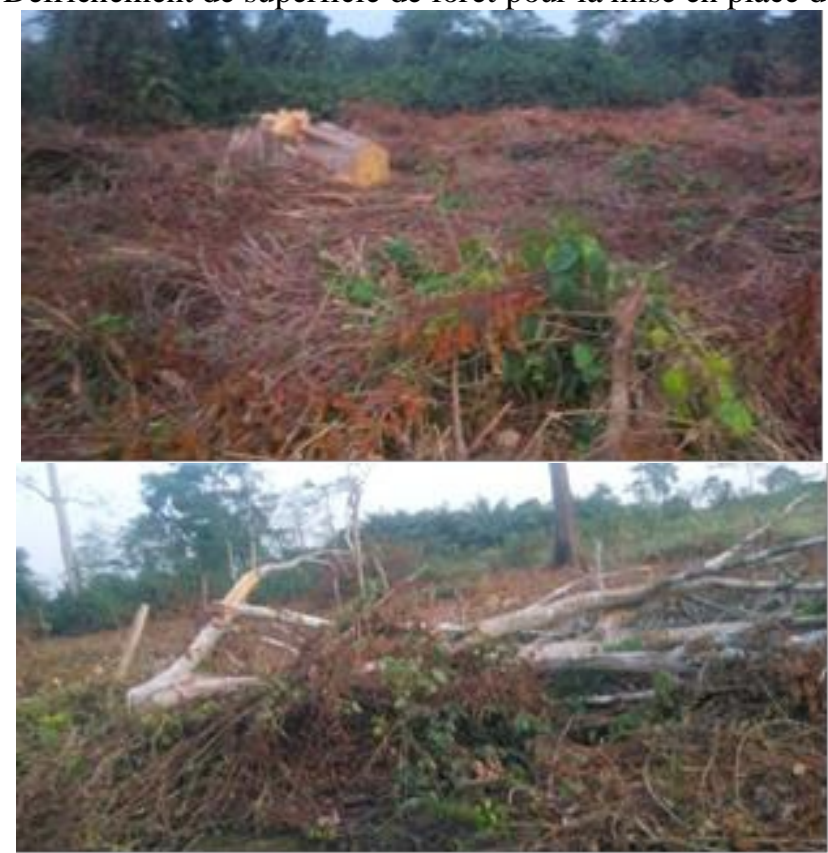

Source: Donnée de l'enquête de terrain, novembre 2017- février 2018

Ici, si certains utilisent cette stratégie pour préserver leur parcelle de forêt dans le seul but de léguer plus tard ces lopins à leurs descendants. D'autres font les cultures sous-bois pour préserver un peu le coté naturel de la forêt. C'est ce qu'affirme un paysan :

"Quand on parle de plantation, ça ne veut pas dire qu'il faut tout couper. Il faut aussi laisser des arbres dans ta plantation, ça te permet de garder aussi la forêt».

En ce qui concerne les forêts villageoises (sacrées) qui sont des patrimoines forestiers naturels (espaces des ancêtres), elles sont des espaces boisés, sacrés, craints et vénérés, réservés à l'expression culturelle d'une communauté donnée. L'accès, la préservation et la gestion sont réglementés par les pouvoirs traditionnels. En effet, ce sont des lieux qui sont perçus comme gage d'identité culturelle et de protection de l'environnement (socle d'un développement harmonieux et durable). Le discours d'un notable est révélateur de cette réalité : 
"La forêt sacrée est notre héritage, c'est là que vivent l'esprit de nos ancêtres. On peut même dire qu'elle fait partie de nous, de notre culture et notre identité, nous ne sommes rien sans elle. C'est un devoir pour nous de ne pas la détruire ».

La forêt incarne donc l'existence sur terre les ancêtres des sociétés africaines. Cet identifiant culturel d'une part joue un rôle de régulation dans les rapports entre les acteurs d'une même famille ou clan et d'autre part dans les rapports entre les populations elles-mêmes.

Les populations de bases de ce Département se reconnaissent à travers ces forêts sacrées. Pour elles, les forêts villageoises ancestrales dites sacrées contribuent au bon fonctionnement de la société du peuple Akyé. C'est ainsi que les autorités villageoises et administratives ont mis en place des stratégies pour préserver la forêt classée de N'dabo située dans le Département d'Akoupé précisément dans le village d'Afféry. En effet, depuis le 18 août 2014, la chefferie traditionnelle de la localité appuyée par le conseil municipal a pris un arrêté portant interdiction de chasse et de toutes autres activités sur le périmètre du Mont N'dabo. Cela se remarque dans les dires d'un chef coutumier en ces termes :

"Nous n'acceptons aucune activité dans nos forêts sacrées, parce que c'est là que vivent nos ancêtres. On ne joue pas avec ça. On veille à ce qu'elle soit conservée ».

Au regard de ces propos, il est formellement interdit à tout individu de construire une habitation et de pratiquer d'autres activités sur un rayon de trente mètres autour de la forêt "N'dabo" vu que le périmètre est classé patrimoine sacré du Département d'Akoupé. Toute infraction aux présentes prescriptions constitue une contravention passible connue telle d'une amende de 100.000 FCFA (153 EURO ou 222 Dollar US) pour les sacrifices d'usage. La chefferie traditionnelle, le secrétaire général de la mairie et les chefs des services municipaux sont chargés de l'exécution du présent arrêté. Cette décision est approuvée et acceptée par toute la population locale y compris les autorités administratives du Département d'Akoupé. Au-delà de l'arrêté qui a été pris par les autorités villageoises et administratives, des jeunes du village d'Afféry ont été désignés pour veiller sur la forêt sacrée. Leur rôle est $\mathrm{d}$ 'interpeler tous ceux qui veulent contourner les règles et normes établies dans le cadre de la préservation de ce patrimoine sacré. C'est ce qui ressort dans les dires du chef de terre :

"Nous avons désigné certains jeunes du village pour veiller sur la forêt sacrée au cas où certains récalcitrants veulent contourner les lois ou passer derrière nous pour s'introduire dans la forêt sacrée, qu'ils les interpellent». 
Photo 2. Une vue de forêt dans le département d'Akoupé

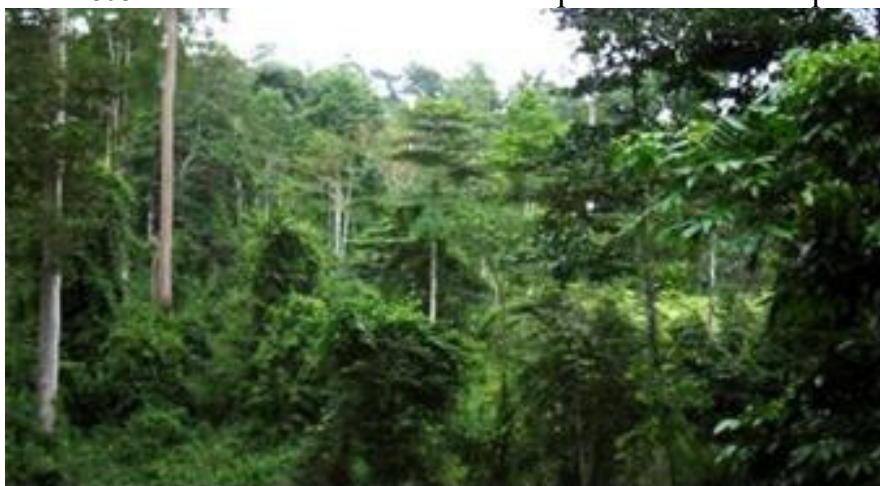

Source: Donnée de l'enquête de terrain, novembre 2017- février 2018

\section{Discussion}

\section{Politiques et stratégies de l'Etat dans la préservation des forêts}

Pour accompagner les populations villageoises dans la préservation, la conservation et la gestion des ressources périssables dont la forêt, l'Etat de Côte d'Ivoire a décidé de mener des actions telles que la sensibilisation et l'éducation environnementale des acteurs du domaine rural du département d'Akoupé. Toutes ces actions sont réalisées à travers les structures étatiques en présence chargées de rendre les agriculteurs plus professionnels. Cette philosophie de la préservation des forêts ressort dans les travaux de Geny et $a l$. (1992) quand ils soutiennent que pour éradiquer le problème de dégradation des ressources, les populations doivent être éduquées et sensibilisées à la gestion de l'environnement et à une gestion raisonnée des ressources naturelles. Ils pensent que cette approche est une solution fiable pour réduire le phénomène de dégradation des forêts.

Ainsi, de nombreuses stratégies de préservation de la forêt sont élaborées dans le département d'Akoupé telles que le reboisement associé à l'adoption des foyers améliorés pour aboutir à une gestion durable des forêts. Ces mêmes idées sont attestées dans les études de Mercier (1991) lorsqu'il évoque le reboisement comme solution possible nécessitant une réelle volonté et des efforts à tous les niveaux. Il soutient que ce reboisement doit être associé à l'adoption des foyers améliorés qui parait pour lui plus pertinent pour atteindre une gestion réelle, durable et efficace des forêts.

Dans cette même veine de solution, Barbier (2004) évoque l'idée de promotion des foyers améliorés; l'utilisation du pétrole, de gaz ou la valorisation de l'énergie solaire qui sont des techniques adaptées. Ces techniques peuvent substituer la demande de bois et réduire la pression exercée sur les arbres et forêts en milieu naturel à des fins énergétiques.

Ce constat est également mis en exergue dans les études de Ozer (2004) quand il dit que la solution à la déforestation consiste en « une réforme 
de la politique énergétique dans les pays concernés ». Cela permettrait de mieux éradiquer le phénomène de disparition des essences et de favoriser également à conserver le peu d'essence que possède encore les pays concernés.

\section{Stratégies développées par les autorités villageoises et administratives du département d'Akoupé dans la préservation des forêts villageoises}

Les forêts tropicales de la Côte d'Ivoire et plus spécifiquement celles du Département d'Akoupé ont toujours fait l'objet de prélèvement de la part des populations locales pour des usages diversifiés tels que (les constructions, l'énergie, les besoins alimentaires humains et animal, la pharmacopée, etc.). Toutefois, la déforestation a débuté dans le département d'Akoupé, dans les années 1960. lorsque l'Etat a mis un programme en place qui encourageait le développement des cultures de rente en zone forestière. Dans cette même perspective, Bongonzini et Lanly (2004) soutiennent que le processus de déforestation en Côte d'Ivoire a surtout été accéléré par la mise en place d'un programme gouvernemental dès 1965. Ce programme encourage le développement des cultures de rente en zone forestière.

Le développement exponentiel des zones de cultures de café et cacao est responsable de nouvelles formes d'appropriation de l'espace, au détriment des zones forestières. Alors, pour sauvegarder les forêts villageoises, les populations locales du département d'Akoupé vont s'établir des règles sousformes de normes qui permettent de préserver leur patrimoine ancestral : forêts villageoises (forêt qui sert à pratiquer l'activité agricole et forêt sacrée). En effet, ces deux types de forêts sont gérés avec des stratégies de préservation qui sont propres aux sociétés traditionnelles. Cette vision traditionnelle de préservation est reprise dans les travaux de Gadou (2001) lorsqu'il affirme que les stratégies d'actions des sociétés traditionnelles qui allaient de la liturgie (prière, sacrifice), à la technologie, en passant par une déontologie codée, spécialisée, mesurée, des sociétés traditionnelles africaines ont assuré la pérennité des sols des climats, des populations de la diversité (faune et flore) jusqu'à la veille de l'économie de traite $\left(15^{\mathrm{e}}\right.$ et $18^{\mathrm{e}}$ siècles $)$.

Les dimensions culturelles interdisant tout individu à une activité quelconque permettent aux populations villageoises d'Akoupé de garder intact l'habitat de leurs ancêtres. La genèse des sociétés africaines permet de mieux cerner les stratégies de préservation des forêts villageoises sacrées afin de les accompagner dans leur intention, celle de garder intact les forêts villageoises. Ce qui est en adéquation avec les résultats des études antérieures de Cherif (2014). L'auteur exige à ce que l'on prenne en compte les connaissances traditionnelles. Pour lui, malgré leur inefficacité dans la situation actuelle du réchauffement global, les connaissances traditionnelles restent d'un apport indéniable dans la préservation de la biodiversité et l'adaptation de 
l'agriculture pluviale au changement climatique. Elles représentent une voie complémentaire à l'adaptation scientifique tout en permettant un échange d'informations entre chercheurs et agriculteurs. Les informations ainsi échangées génèrent de nouvelles stratégies d'adaptation en faisant la promotion de pratiques agricoles innovantes au niveau villageois ivoirien.

\section{Conclusion}

A travers cet article qui traite des stratégies de préservation des forêts villageoises dans le département d'Akoupé au Sud-est de la Côte d'Ivoire, l'objectif est de décrire les stratégies développées par les acteurs dans la préservation de ces forêts villageoises. Ainsi, la démarche s'appuie sur des enquêtes de terrain, une méthode d'analyse spécifique et une perspective théorique indiquée.

Concernant les politiques et stratégies de l'Etat dans la préservation des forêts villageoises, il faut noter que l'Etat de Côte d'Ivoire a pris des engagements avec des institutions internationales telles que le REDD+ dans le but de contribuer à la lutte contre le changement climatique et surtout pour la restauration de sa couverture forestière dégradée. Aussi, l'Etat a confié des tâches aux structures qui exercent dans le domaine agricole afin de contribuer à la promotion du monde rural par la professionnalisation des producteurs. Cela leur permet de détruire moins les parcelles réservées à l'activité agricole.

S'agissant des stratégies développées par les autorités villageoises et administratives dans la préservation des forêts villageoises, il ressort que ces stratégies sont plus axées sur les forêts sacrées que celles qui sont consacrées à l'activité agricole. Des normes traditionnelles sont établies pour préserver ces forêts sacrées en accord avec les autorités administratives locales. Il est établi autour de ces forêts villageoises un système de sanction accepté par tous les acteurs du département d'Akoupé.

En dépit de toutes ces stratégies développées par les acteurs pour la préservation des forêts villageoises dans le département d'Akoupé, mises à la lumière par les résultats de cette étude, il parait nécessaire pour l'Etat d'étendre ses compétences sur l'ensemble des forêts nationales. Ce qui suppose de ne pas se limiter au contrôle de l'intégrité des espaces forestiers, mais de règlementer aussi les pratiques sylvicoles. Enfin, l'appel à des actions de plus en plus coordonnées des acteurs du domaine forestier relativement à l'éradication du phénomène de disparition des forêts villageoises se fait avec persistance.

\section{References:}

1. Allanby,M. (1993). The MacMillan Dictionary of the environment. Londres, MacMillan, cité par TRA, F (2007) pages 5052. 
2. Banque,M. (2013). Douane française et ivoirienne, citées par Business France 2013 et 2014, IGN, MAAF, pages 18.

3. Barbier, C. (2004). Désertification et forêt, Silva Reat, Laballery clamacy (58), 159pages.

4. Bongonzini, J.C. \& Lanly, JP. (2004). Les forêts tropicales, CIRAD, ed karthala, 164pages.

5. Chatelain, C, Dao, H. \& Spichiger, R. (2004). Forest cover changes in Côte d'Ivoire and Upper Guinea. Biodiversity of West Africa forest, an Ecological Atlas of Woody plants, Species, Cabi publishing, Cambuge (UK) pages 15-32.

6. CNUD (1992). Principes 3 de la déclaration sur les forêts, Rio De Janeiro, p3.

7. Christopher, E. (1996). Paradigmes de conservation des forêts, Unasylva, 187, vol 47: pages 3-8.

8. Ezzine Detruiz, P. (2008). Prospects for reduced impact logging in central African logging consessious, forest ecology and management, 256, pages 1509-1516.

9. Gadou, D. (2001). La préservation de la biodiversité : les réponses de la tradition religieuse africaine, In : The African anthropologist, Vol. $8, \mathrm{n}^{\circ} 2$ : pages $178-199$.

10. Geny, P. et al. (1992). Environnement et développement rural, guide de gestion des ressources naturelles, Frison-Roche, Saint-Etienne 14, 18pages.

11. Ibo, G. (1993). La politique coloniale de protection de la nature de 1900 à 1958 : revue française et histoire d'Outre-Mer, 1993, (298), pages 83-104.

12. Ibo, G. (2005). Les nouvelles orientations de la gestion du patrimoine naturel en Côte d'Ivoire, IRD, Edition, 2005, pages 71-96.

13. Mead, G. (1934). L'esprit, le soi et la société, Nouvelle tradition et introduction par Daniel Cefai et Louis Quéré, Paris PUF, Collection le lien social, 2006, 434 pages.

14. Mercier, J.R. (1991). La déforestation en Afrique, Situation perspectives, Aix-en-Provence, Edi sud, 176pages.

15. Ministère de l'Agriculture et des Ressources Animales (1993). Charte pour la réhabilitation du domaine forestier de l'Etat, 118pages.

16. Ozer, P. (2004). Bois de feu et déboisement au Sahel mise au point, Sécheresse n³, vol 15, septembre 2004 pages 243-251.

17. Rapport annuel de la Direction Régionale du Ministère de l'Agriculture et des Ressources Animales d'Adzopé sur les cultures vivrières et pérennes (2015-2016). 22 pages. 
18. Rapport de l'étude sur l'exploitation forestière et les contraintes d'une gestion durable des forêts dans le domaine rural en Côte d'Ivoire, (2013). Cirad, Louppe D. et N'klo O.

19. Rapport de projet, BNETD (2005). Bilan de la politique forestière en Côte d'Ivoire et propositions d'orientations, de principes et de règle de fonctionnement du secteur. BNETD, 2005, Abidjan, pages 7-15.

20. Rapport (2019). Sur la stratégie ivoirienne de la lutte contre la déforestation dans la cacaoculture présenté au SIA, pages 2-5.

21. Sadia, C. (2014). Construire la résilience au changement climatique par les connaissances locales : le cas des régions montagneuses et de savanes de Côte d'Ivoire, 28pages.

22. Tra, F. (2007). La problématique de la conservation du Parc national de la Marahoué, Thèse de doctorat unique en sociologie de l'environnement, Université Félix Houphouët-Boigny, AbidjanCocody (CI), 474pages. 